\title{
ASYMPTOTIC BEHAVIOR OF SOLUTIONS OF NONLINEAR NEUTRAL DELAY DIFFERENTIAL EQUATIONS
}

\author{
GENGPING WEI
}

\begin{abstract}
This paper is concerned with the nonlinear neutral delay differential equation with positive and negative coefficients

$$
[x(t)-c(t) x(t-\tau)]^{\prime}+p(t) f(x(t-\delta))-q(t) f(x(t-\sigma))=0, t \geq t_{0},
$$

where $\tau \in(0, \infty), \delta$ and $\sigma \in[0, \infty), c(t) \in C\left(\left[t_{0}, \infty\right), R\right), p(t)$ and $q(t) \in C\left(\left[t_{0}, \infty\right),[0, \infty)\right)$, $f \in C(R, R)$. Sufficient conditions are obtained under which every solution of the above equation is bounded and tends to a constant as $t \rightarrow \infty$. Our results extend and improve some known results.
\end{abstract}

\section{Introduction}

Consider the following nonlinear neutral delay differential equation with positive and negative coefficients

$$
[x(t)-c(t) x(t-\tau)]^{\prime}+p(t) f(x(t-\delta))-q(t) f(x(t-\sigma))=0, t \geq t_{0},
$$

where $\tau \in(0, \infty), \delta$ and $\sigma \in[0, \infty), c(t) \in C^{1}\left(\left[t_{0}, \infty\right), R\right), p(t)$ and $q(t) \in C\left(\left[t_{0}, \infty\right),[0, \infty)\right), f \in$ $C(R, R)$. We note that when $f(x) \equiv x$, equation (1) reduces to the linear differential equation

$$
[x(t)-c(t) x(t-\tau)]^{\prime}+p(t) x(t-\delta)-q(t) x(t-\sigma)=0, \quad t \geq t_{0} .
$$

The asymptotic behavior of solutions of linear equation (2) and its special cases, i.e., $c(t) \equiv 0$ or $q(t) \equiv 0$ or $c(t) \equiv 0$ and $q(t) \equiv 0$, has been studied by several authors, see, for example, [1]- [7]. In [1], the authors studied the asymptotic behavior of equation (2) with $c(t) \equiv 0$ and $q(t) \equiv 0$, i.e.,

$$
x^{\prime}(t)+p(t) x(t-\delta)=0, \quad t \geq t_{0}
$$

Received August 5, 2012, accepted October 4, 2013.

2010 Mathematics Subject Classification. 34K20, 34K25, 34K40.

Key words and phrases. Neutral delay differential equation, boundedness, asymptotic behavior, Liapunov functional. 
and obtained that if $p(t) \in C\left(\left[t_{0}, \infty\right),(0, \infty)\right)$ and $\delta \geq 0$ are such that

$$
\int_{t_{0}}^{\infty} p(s) d s=\infty
$$

and

$$
\limsup _{t \rightarrow \infty} \int_{t-\delta}^{t} p(s+\delta) d s<1,
$$

then every solution of Eq.(3) tends to zero as $t \rightarrow \infty$. In [2,3], it is proved by constructing an effective Liapunov functional that if $p(t) \in C\left(\left[t_{0}, \infty\right),(0, \infty)\right)$ and $\delta \geq 0$ then

$$
\limsup _{t \rightarrow \infty} \int_{t-\delta}^{t+\delta} p(s+\delta) d s<2
$$

together with (4) implies that every solution of Eq.(3) tends to zero as $t \rightarrow \infty$. While in [4, 5], by employing a non-Liapunov approach, the authors studied the stability of equation (3) and obtained another result, which states that if $p(t) \in C\left(\left[t_{0}, \infty\right),(0, \infty)\right)$ and $\delta \geq 0$, then

$$
\limsup _{t \rightarrow \infty} \int_{t-\delta}^{t} p(s) d s<\frac{3}{2}
$$

together with (4) implies that every solution of Eq.(3) tends to zero as $t \rightarrow \infty$. Obviously conditions (6) and (7) are weaker than condition (5); also note that it is shown in [9] that conditions (6) and (7) are independent. Recently, the asymptotic behavior of some special cases of nonlinear equation (1), i.e., $c(t) \equiv 0$ or $q(t) \equiv 0$ or $c(t) \equiv 0$ and $q(t) \equiv 0$, is considered under some strong restriction on $f$ in [8]-[11]. The purpose of this paper is to consider the more general Eq.(1) and to derive sufficient conditions under which every solution of Eq.(1) is bounded and tends to a constant as $t \rightarrow \infty$. As special cases mentioned above, our results relax the restriction on $f$ in $[9,11]$; in the linear cases of $f(x) \equiv x$, our results include the results in [3] and improve the related results in $[1,6]$.

Let $\rho=\max \{\tau, \delta, \sigma\}$. By a solution of Eq.(1) we mean a function $x(t)$ which is defined for $t \geq t_{0}-\rho$ and satisfies Eq.(1) for $t \geq t_{0}$. It is easy to see that for any given $t_{0}$ and initial conditions of the form $x\left(t_{0}+s\right)=\varphi(s), s \in[-\rho, 0]$, Eq.(1) has an unique solution $x(t)$ which is defined for $t \geq t_{0}-\rho$ and satisfies the above initial conditions.

As is customary, a solution of (1) or (2) is said to be nonoscillatory if it is eventually positive or eventually negative. Otherwise, it will be called oscillatory. For the general background on functional differential equations, one can refer to [12, 13].

\section{Main results}

In connection with the nonlinear function $f$, we assume that

(H) There are constants $L>0$ and $M>0$ such that

$$
x f(x)>0 \text {, for } x \in R, x \neq 0 \text { and } L=\inf \left\{\frac{f(x)}{x} \| x \mid>0\right\}, M=\sup \left\{\frac{f(x)}{x} \| x \mid>0\right\} .
$$


Theorem 1. Let $(\mathrm{H})$ hold. Assume that

$$
\begin{gathered}
\delta \geq \sigma \\
p^{*}(t)=p(t)-q(t+\sigma-\delta)>0 \text { for } t \geq t_{1}=t_{0}+\delta-\sigma ; \\
\limsup _{t \rightarrow \infty}\left[|c(t)|+M \int_{t-\delta}^{t-\sigma} q(s+\sigma) d s\right]=\mu<1 \\
\limsup _{t \rightarrow \infty}\left[\mu\left(1+\frac{p^{*}(t+\tau+\delta)}{L^{2} p^{*}(t+\delta)}\right)+\int_{t-\delta}^{t+\delta} p^{*}(s+\delta) d s+\frac{q(t+\sigma)}{p^{*}(t+\delta)} \int_{t-\delta}^{t} p^{*}(s+2 \delta) d s\right]<\frac{2}{M} .
\end{gathered}
$$

Then every solution of (1) is bounded.

Proof. Let $x(t)$ be any solution of (1). We shall prove that $x(t)$ is bounded. For this purpose, we rewrite (1) in the form

$$
\begin{aligned}
{\left[x(t)-c(t) x(t-\tau)-\int_{t-\delta}^{t-\sigma} q(s+\sigma) f(x(s)) d s-\int_{t-\delta}^{t}\right.} & \left.p^{*}(s+\delta) f(x(s)) d s\right]^{\prime} \\
& +p^{*}(t+\delta) f(x(t))=0, t \geq t_{1} .
\end{aligned}
$$

From (10) and (11), we can select an $\varepsilon>0$ sufficiently small such that $\mu+\varepsilon<1$ and

$$
\limsup _{t \rightarrow \infty}\left[(\mu+\varepsilon)\left(1+\frac{p^{*}(t+\tau+\delta)}{L^{2} p^{*}(t+\delta)}\right)+\int_{t-\delta}^{t+\delta} p^{*}(s+\delta) d s \frac{q(t+\sigma)}{p^{*}(t+\delta)} \int_{t-\delta}^{t} p^{*}(s+2 \delta) d s\right]<\frac{2}{M}
$$

also, we can select sufficiently large $t^{*}>t_{0}$ such that

$$
|c(t)|+M \int_{t-\delta}^{t-\sigma} q(s+\sigma) d s \leq \mu+\varepsilon, \text { for } t \geq t^{*}
$$

noting $(\mathrm{H})$ and (14), we have

$$
|c(t)| x^{2}(t-\tau) \leq \frac{\mu+\varepsilon}{L^{2}} f^{2}(x(t-\tau)), \quad t \geq t^{*} .
$$

Now we introduce three functionals as

$$
\begin{aligned}
& V_{1}(t)=\left[x(t)-c(t) x(t-\tau)-\int_{t-\delta}^{t-\sigma} q(s+\sigma) f(x(s)) d s-\int_{t-\delta}^{t} p^{*}(s+\delta) f(x(s)) d s\right]^{2}, t \geq t_{1}, \\
& V_{2}(t)=\int_{t-\delta}^{t} p^{*}(s+2 \delta) \int_{s}^{t} p^{*}(u+\delta) f^{2}(x(u)) d u d s, t \geq t_{1}, \\
& V_{3}(t)=\int_{t-\delta}^{t} p^{*}(s+2 \delta) \int_{s}^{t} q(u+\sigma) f^{2}(x(u)) d u d s+\frac{\mu+\varepsilon}{L^{2}} \int_{t-\tau}^{t} p^{*}(s+\tau+\delta) f^{2}(x(s)) d s, t \geq t_{1} .
\end{aligned}
$$

In what follows, for the sake of convenience, when we write a functional inequality without specifying its domain of validity, we mean that it holds for all sufficiently large $t$.

Calculating $\frac{d V_{1}(t)}{d t}, \frac{d V_{2}(t)}{d t}$ and $\frac{d V_{3}(t)}{d t}$ along the solution of (1), we have

$$
\frac{d V_{1}(t)}{d t}=-p^{*}(t+\delta)[2 x(t) f(x(t))-2 c(t) x(t-\tau) f(x(t))
$$




$$
\begin{aligned}
& \left.-\int_{t-\delta}^{t-\sigma} q(s+\sigma)(2 f(x(t)) f(x(s))) d s-\int_{t-\delta}^{t} p^{*}(s+\delta)(2 f(x(t)) f(x(s))) d s\right] \\
\leq & -p^{*}(t+\delta)\left[2 x(t) f(x(t))-|c(t)| x^{2}(t-\tau)-|c(t)| f^{2}(x(t))\right. \\
& -f^{2}(x(t)) \int_{t-\delta}^{t-\sigma} q(s+\sigma) d s-\int_{t-\delta}^{t-\sigma} q(s+\sigma) f^{2}(x(s)) d s \\
& \left.-f^{2}(x(t)) \int_{t-\delta}^{t} p^{*}(s+\delta) d s-\int_{t-\delta}^{t} p^{*}(s+\delta) f^{2}(x(s)) d s\right], \\
\frac{d V_{2}(t)}{d t}= & -p^{*}(t+\delta) \int_{t-\delta}^{t} p^{*}(s+\delta) f^{2}(x(s)) d s+p^{*}(t+\delta) f^{2}(x(t)) \int_{t-\delta}^{t} p^{*}(s+2 \delta) d s,
\end{aligned}
$$

and

$$
\begin{aligned}
\frac{V_{3}(t)}{d t}= & q(t+\sigma) f^{2}(x(t)) \int_{t-\delta}^{t} p^{*}(s+2 \delta) d s-p^{*}(t+\delta) \int_{t-\delta}^{t} q(s+\sigma) f^{2}(x(s)) d s \\
& +\frac{\mu+\varepsilon}{L^{2}} p^{*}(t+\tau+\delta) f^{2}(x(t))-\frac{\mu+\varepsilon}{L^{2}} p^{*}(t+\delta) f^{2}(x(t-\tau)) \\
\leq & q(t+\sigma) f^{2}(x(t)) \int_{t-\delta}^{t} p^{*}(s+2 \delta) d s-p^{*}(t+\delta) \int_{t-\delta}^{t-\sigma} q(s+\sigma) f^{2}(x(s)) d s \\
& +\frac{\mu+\varepsilon}{L^{2}} p^{*}(t+\tau+\delta) f^{2}(x(t))-\frac{\mu+\varepsilon}{L^{2}} p^{*}(t+\delta) f^{2}(x(t-\tau)) .
\end{aligned}
$$

Set $V(t)=V_{1}(t)+V_{2}(t)+V_{3}(t)$. By (19)-(21), (14) and (15), we get

$$
\begin{aligned}
\frac{V(t)}{d t}= & \frac{V_{1}(t)}{d t}+\frac{V_{2}(t)}{d t}+\frac{V_{3}(t)}{d t} \\
\leq & -p^{*}(t+\delta)\left[2 x(t) f(x(t))-|c(t)| x^{2}(t-\tau)-|c(t)| f^{2}(x(t))\right. \\
& -\int_{t-\delta}^{t-\sigma} q(s+\sigma) f^{2}(x(s)) d s-f^{2}(x(t)) \int_{t-\delta}^{t-\sigma} q(s+\sigma) d s \\
& -\int_{t-\delta}^{t} p^{*}(s+\delta) f^{2}(x(s)) d s-f^{2}(x(t)) \int_{t-\delta}^{t} p^{*}(s+\delta) d s \\
& +\int_{t-\delta}^{t} p^{*}(s+\delta) f^{2}(x(s)) d s-f^{2}(x(t)) \int_{t-\delta}^{t} p^{*}(s+2 \delta) d s \\
& -f^{2}(x(t)) \frac{q(t+\sigma)}{p^{*}(t+\delta)} \int_{t-\delta}^{t} p^{*}(s+2 \delta) d s+\int_{t-\delta}^{t-\sigma} q(s+\sigma) f^{2}(x(s)) d s \\
& \left.-\frac{\mu+\varepsilon}{L^{2}} f^{2}(x(t)) \frac{p^{*}(t+\tau+\delta)}{p^{*}(t+\delta)}+\frac{\mu+\varepsilon}{L^{2}} f^{2}(x(t-\tau))\right] \\
\leq & -p^{*}(t+\delta) f^{2}(x(t))\left[\frac{2 x(t)}{f(x(t))}-\left(|c(t)|+\int_{t-\delta}^{t-\sigma} q(s+\sigma) d s\right)\right. \\
& \left.-\int_{t-\delta}^{t+\delta} p^{*}(s+\delta) d s-\frac{q(t+\sigma)}{p^{*}(t+\delta)} \int_{t-\delta}^{t} p^{*}(s+2 \delta) d s-\frac{\mu+\varepsilon}{L^{2}} \frac{p^{*}(t+\tau+\delta)}{p^{*}(t+\delta)}\right] \\
\leq & -p^{*}(t+\delta) f^{2}(x(t))\left[\frac{2 x(t)}{f(x(t))}-(\mu+\varepsilon)\left(1+\frac{p^{*}(t+\tau+\delta)}{L^{2} p^{*}(t+\delta)}\right)\right. \\
& \left.-\frac{q(t+\sigma)}{p^{*}(t+\delta)} \int_{t-\delta}^{t} p^{*}(s+2 \delta) d s-\int_{t-\delta}^{t+\delta} p^{*}(s+\delta) d s\right] \\
\leq & -p^{*}(t+\delta) f^{2}(x(t))\left\{\frac{2}{M}-\left[(\mu+\varepsilon)\left(1+\frac{p^{*}(t+\tau+\delta)}{L^{2} p^{*}(t+\delta)}\right)\right.\right. \\
&
\end{aligned}
$$




$$
\left.\left.+\frac{q(t+\sigma)}{p^{*}(t+\delta)} \int_{t-\delta}^{t} p^{*}(s+2 \delta) d s+\int_{t-\delta}^{t+\delta} p^{*}(s+\delta) d s\right]\right\},
$$

which, together with (13), implies

$$
\int_{t_{1}}^{\infty} p^{*}(t+\delta) f^{2}(x(t)) d s<\infty
$$

and, hence, for any $r>0$ we have

$$
\lim _{t \rightarrow \infty} \int_{t-r}^{t} p^{*}(s+\delta) f^{2}(x(s)) d s=0
$$

Noting (13), we have

$$
\begin{aligned}
0 \leq V_{2}(t) & \leq \int_{t-\delta}^{t} p^{*}(s+2 \delta) \int_{t-\delta}^{t} p^{*}(u+\delta) f^{2}(x(u)) d u d s \\
& =\int_{t}^{t+\delta} p^{*}(s+\delta) d s \cdot \int_{t-\delta}^{t} p^{*}(u+\delta) f^{2}(x(u)) d u \\
& \leq \frac{2}{M} \int_{t-\delta}^{t} p^{*}(u+\delta) f^{2}(x(u)) d u
\end{aligned}
$$

and

$$
\begin{aligned}
0 \leq V_{3}(t)= & \int_{t-\delta}^{t}\left(p^{*}(s+2 \delta) \int_{s}^{t} q(u+\sigma) f^{2}(x(u)) d u\right) d s \\
& +\frac{(\mu+\varepsilon)}{L^{2}} \int_{t-\tau}^{t} p^{*}(s+\tau+\delta) f^{2}(x(s)) d s \\
\leq & \int_{t-\delta}^{t}\left(q(u+\sigma) \int_{t-\delta}^{u} p^{*}(s+2 \delta) d s\right) f^{2}(x(u)) d u \\
& +\frac{2}{M} \int_{t-\tau}^{t} p^{*}(s+\delta) f^{2}(x(s)) d s \\
\leq & \int_{t-\delta}^{t}\left(q(u+\sigma) \int_{u-\delta}^{u} p^{*}(s+2 \delta) d s\right) f^{2}(x(u)) d u \\
& +\frac{2}{M} \int_{t-\tau}^{t} p^{*}(s+\delta) f^{2}(x(s)) d s \\
\leq & \int_{t-\delta}^{t} \frac{2}{M} p^{*}(u+\delta) f^{2}(x(u)) d u+\frac{2}{M} \int_{t-\tau}^{t} p^{*}(s+\delta) f^{2}(x(s)) d s .
\end{aligned}
$$

(25) and (26) together with (24) imply $\lim _{t \rightarrow \infty} V_{2}(t)=0$ and $\lim _{t \rightarrow \infty} V_{3}(t)=0$. On the other hand, by (13) and (22), we see that $V(t)$ is eventually decreasing. In view of $V(t) \geq 0$, the limit $\lim _{t \rightarrow \infty} V(t)=\alpha$ exists and is finite, and thus, $\lim _{t \rightarrow \infty} V(t)=\lim _{t \rightarrow \infty} V_{1}(t)=\alpha$; that is,

$$
\lim _{t \rightarrow \infty}\left[x(t)-c(t) x(t-\tau)-\int_{t-\delta}^{t-\sigma} q(s+\sigma) f(x(s)) d s-\int_{t-\delta}^{t} p^{*}(s+\delta) f(x(s)) d s\right]^{2}=\alpha .
$$

Let

$$
y(t)=x(t)-c(t) x(t-\tau)-\int_{t-\delta}^{t-\sigma} q(s+\sigma) f(x(s)) d s-\int_{t-\delta}^{t} p^{*}(s+\delta) f(x(s)) d s,
$$


then

$$
y^{\prime}(t)+p^{*}(t+\delta) f(x(t))=0, t \geq t_{1}
$$

and

$$
\lim _{t \rightarrow \infty} y^{2}(t)=\alpha .
$$

If $\alpha=0$, then $\lim _{t \rightarrow \infty} y(t)=0$. If $\alpha>0$, noting that $y(t)$ is continuous, then $y(t)$ is eventually positive or eventually negative. So,

$$
\lim _{t \rightarrow \infty} y(t)=\beta
$$

that is,

$$
\begin{aligned}
\lim _{t \rightarrow \infty} y(t)= & \lim _{t \rightarrow \infty}\left[x(t)-c(t) x(t-\tau)-\int_{t-\delta}^{t-\sigma} q(s+\sigma) f(x(s)) d s\right. \\
& \left.-\int_{t-\delta}^{t} p^{*}(s+\delta) f(x(s)) d s\right]=\beta,
\end{aligned}
$$

where $\beta=\sqrt{\alpha}$ or $\beta=-\sqrt{\alpha}$. In view of (28), we have

$$
\int_{t-\delta}^{t} p^{*}(s+\delta) f(x(s)) d s=y(t-\delta)-y(t) .
$$

So,

$$
\lim _{t \rightarrow \infty} \int_{t-\delta}^{t} p^{*}(s+\delta) f(x(s)) d s=0 .
$$

By (29) and (30), we have

$$
\lim _{t \rightarrow \infty}\left[x(t)-c(t) x(t-\tau)-\int_{t-\delta}^{t-\sigma} q(s+\sigma) f(x(s)) d s\right]=\beta .
$$

Next, we shall show $|x(t)|$ is bounded. In fact, if $|x(t)|$ is unbounded, then there exists a sequence $\left\{r_{n}\right\}$ such that $r_{n} \rightarrow \infty,\left|x\left(r_{n}\right)\right| \rightarrow \infty$ as $n \rightarrow \infty$ and

$$
\left|x\left(r_{n}\right)\right|=\sup _{t_{0} \leq t \leq r_{n}}|x(t)|
$$

Thus, noticing (14), (32), we have

$$
\begin{aligned}
& \left|x\left(r_{n}\right)-c\left(r_{n}\right) x\left(r_{n}-\tau\right)-\int_{r_{n}-\delta}^{r_{n}-\sigma} q(s+\sigma) f(x(s)) d s\right| \\
& \quad \geq\left|x\left(r_{n}\right)\right|-\left|c\left(r_{n}\right)\right|\left|x\left(r_{n}-\tau\right)\right|-\int_{r_{n}-\delta}^{r_{n}-\sigma} q(s+\sigma) M|x(s)| d s \\
& \quad \geq\left|x\left(r_{n}\right)\right|\left(1-\left|c\left(r_{n}\right)\right|-M \int_{r_{n}-\delta}^{r_{n}-\sigma} q(s+\sigma) d s\right) \\
& \quad \geq\left|x\left(r_{n}\right)\right|(1-\mu-\varepsilon) \rightarrow \infty \text { as } n \rightarrow \infty,
\end{aligned}
$$

which contradicts (31). So $|x(t)|$ is bounded. The proof of Theorem 1 is complete.

Now we study asymptotic behavior of solutions of (1). 
Theorem 2. Let $(\mathrm{H})$, (8) and (9) hold. Assume that $c(t) \geq 0$ or $c(t) \leq 0$ for sufficiently large $t$, i.e., $c(t)$ does not change sign for sufficiently large $t$, and

$$
\begin{gathered}
\lim _{t \rightarrow \infty}|c(t)|=c<1 \\
\lim _{t \rightarrow \infty} \int_{t-\delta}^{t-\sigma} q(s+\sigma) d s=0 ; \\
\limsup _{t \rightarrow \infty}\left[c\left(1+\frac{p^{*}(t+\tau+\delta)}{L^{2} p^{*}(t+\delta)}\right)+\int_{t-\delta}^{t+\delta} p^{*}(s+\delta) d s+\frac{q(t+\sigma)}{p^{*}(t+\delta)} \int_{t-\delta}^{t} p^{*}(s+2 \delta) d s\right]<\frac{2}{M} .
\end{gathered}
$$

Then every solution of (1) tends to a constant as $t \rightarrow \infty$.

Proof. Let $x(t)$ be any solution of (1). (33) and (34) imply that

$$
\limsup _{t \rightarrow \infty}\left[|c(t)|+M \int_{t-\delta}^{t-\sigma} q(s+\sigma) d s\right]=c<1 .
$$

So, from the proof of Theorem 1 we know that $|x(t)|$ is bounded and (31) holds. Noticing condition (34), we obtain

$$
\begin{aligned}
0 & \leq\left|\int_{t-\delta}^{t-\sigma} q(s+\sigma) f(x(s)) d s\right| \leq \int_{t-\delta}^{t-\sigma} q(s+\sigma)|f(x(s))| d s \\
& \leq M \int_{t-\delta}^{t-\sigma} q(s+\sigma)|x(s)| d s \rightarrow 0 \text { as } t \rightarrow \infty
\end{aligned}
$$

which, together with (31), implies that

$$
\lim _{t \rightarrow \infty}[x(t)-c(t) x(t-\tau)]=\beta .
$$

Next, we shall prove that

$$
\lim _{t \rightarrow \infty} x(t) \text { exists and is finite. }
$$

If $c=0$, clearly $\lim _{t \rightarrow \infty} x(t)=\beta$, which shows (37) holds. If $0<c<1$, let

$$
\limsup _{t \rightarrow \infty} x(t)=\omega_{1}, \quad \liminf _{t \rightarrow \infty} x(t)=\omega_{2}
$$

and let $\left\{u_{i}\right\}$ and $\left\{v_{i}\right\}$ be two sequences such that $u_{i} \rightarrow \infty, v_{i} \rightarrow \infty$ as $i \rightarrow \infty$, and

$$
\lim _{i \rightarrow \infty} x\left(u_{i}\right)=\omega_{1}, \quad \lim _{i \rightarrow \infty} x\left(v_{i}\right)=\omega_{2} .
$$

For sufficiently large $t_{2}$, we consider the following two possible cases:

Case 1. $-1<c(t) \leq 0$ for $t>t_{2}$, we have

$$
\beta=\lim _{i \rightarrow \infty}\left[x\left(u_{i}\right)-c\left(u_{i}\right) x\left(u_{i}-\tau\right)\right]=\omega_{1}+c \lim _{i \rightarrow \infty} x\left(u_{i}-\tau\right) \geq \omega_{1}+c \omega_{2},
$$

and 


$$
\beta=\lim _{i \rightarrow \infty}\left[x\left(v_{i}\right)-c\left(v_{i}\right) x\left(v_{i}-m\right)\right]=\omega_{2}+c \lim _{i \rightarrow \infty} x\left(v_{i}-\tau\right) \leq \omega_{2}+c \omega_{1} .
$$

Thus, $0 \leq \omega_{1}-\omega_{2} \leq c\left(\omega_{1}-\omega_{2}\right)$, so that $\omega_{1}=\omega_{2}=\frac{\beta}{1+c}$, which shows (37) holds.

Case 2. $0 \leq c(t)<1$ for $t>t_{2}$, we have

and

$$
\begin{aligned}
\omega_{1}=\lim _{i \rightarrow \infty} x\left(u_{i}\right) & =\lim _{i \rightarrow \infty}\left[x\left(u_{i}\right)-c\left(u_{i}\right) x\left(u_{i}-\tau\right)+c\left(u_{i}\right) x\left(u_{i}-\tau\right)\right] \\
& =\beta+c \lim _{i \rightarrow \infty} x\left(u_{i}-\tau\right) \leq \beta+c \omega_{1},
\end{aligned}
$$

$$
\begin{aligned}
\omega_{2}=\lim _{i \rightarrow \infty} x\left(v_{i}\right) & =\lim _{i \rightarrow \infty}\left[x\left(v_{i}\right)-c\left(v_{i}\right) x\left(v_{i}-\tau\right)+c\left(v_{i}\right) x\left(v_{i}-\tau\right)\right] \\
& =\beta+c \lim _{i \rightarrow \infty} x\left(v_{i}-\tau\right) \geq \beta+c \omega_{2}
\end{aligned}
$$

Thus, $\omega_{1} \leq \frac{\beta}{1-c} \leq \omega_{2}$, which together with $\omega_{1} \geq \omega_{2}$ implies $\omega_{1}=\omega_{2}=\frac{\beta}{1-c}$, so that (37) holds. The proof of Theorem 2 is complete.

By Theorem 2, we have the following asymptotic behavior result immediately.

Theorem 3. The conditions of Theorem 1 imply that every oscillatory solution of (1) tends to zero as $t \rightarrow \infty$.

Theorem 4. The conditions in Theorem 2 together with

$$
\int_{t_{0}}^{\infty} p^{*}(t) d s=\infty
$$

imply that every solution of (1) tends to zero as $t \rightarrow \infty$.

Proof. Condition (H) implies that for any $\xi>0$ there is $\eta>0$ such that

$$
|f(x)| \geq \eta \text {, for }|x| \geq \xi \text {. }
$$

By theorem 3, we only have to prove that every nonoscillatory solution of (1) tends to zero as $t \rightarrow \infty$. Without loss of generality, let $x(t)$ be an eventually positive solution of (1), we shall prove $\lim _{t \rightarrow \infty} x(t)=0$. As in the proof of Theorem 1, we can rewrite (1) in the form of (28). Integrating from $t_{0}$ to $t$ on both sides of (28) produces

$$
\int_{t_{0}}^{t} p^{*}(s+\delta) f(x(s)) d s=y\left(t_{0}\right)-y(t) .
$$

By using (29) we have

$$
\int_{t_{0}}^{\infty} p^{*}(s+\delta) f(x(s)) d s<\infty
$$

which, together with (38) yields $\liminf _{t \rightarrow \infty} f(x(t))=0$. We claim that

$$
\liminf _{t \rightarrow \infty} x(t)=0
$$


Let $\left\{w_{i}\right\}$ be a integer sequence such that $w_{i} \rightarrow \infty$ as $i \rightarrow \infty$ and $\lim _{i \rightarrow \infty} f\left(x\left(w_{i}\right)\right)=0$. We must have $\liminf _{i \rightarrow \infty} x\left(w_{i}\right)=d=0$. In fact, if $d>0$, then there is a subsequence $\left\{w_{i_{j}}\right\}$ of $\left\{w_{i}\right\}$ such that $x\left(w_{i_{j}}\right) \geq d / 2$ for sufficiently large $j$. By (39) we have $f\left(x\left(w_{i_{j}}\right)\right) \geq \zeta$ for some $\zeta>0$ and sufficiently large $j$, which yields a contradiction because of $\lim _{j \rightarrow \infty} f\left(x\left(w_{i_{j}}\right)\right)=0$. Therefore, (40) holds. On the other hand, by Theorem 2, we have $\lim _{t \rightarrow \infty} x(t)$ exists. Therefore $\lim _{t \rightarrow \infty} x(t)=0$. The proof is complete.

As application of Theorems 2 and 4, taking $f(x) \equiv x$, for the linear equation (2) we have:

Corollary 1. Let $c(t) \geq 0$ or $c(t) \leq 0$ for sufficiently large t. Assume that (8), (9), (33) and (34) hold and

$$
\limsup _{t \rightarrow \infty}\left[c\left(1+\frac{p^{*}(t+\tau+\delta)}{p^{*}(t+\delta)}\right)+\int_{t-\delta}^{t+\delta} p^{*}(s+\delta) d s+\frac{q(t+\sigma)}{p^{*}(t+\delta)} \int_{t-\delta}^{t} p^{*}(s+2 \delta) d s\right]<2
$$

Then every solution of equation (2) tends to a constant as $t \rightarrow \infty$. Furthermore, if (38) holds, then every solution of equation (2) tends to zero as $t \rightarrow \infty$.

In Theorem 4, taking $c(t) \equiv c, q(t) \equiv 0$ and $f(x) \equiv x$, we have:

Corollary 2. Let $|c|<1$. Assume that $\tau \in(0, \infty), \delta \in[0, \infty), p(t) \in C\left(\left[t_{0}, \infty\right),(0, \infty)\right)$. Then

$$
\limsup _{t \rightarrow \infty}\left\{|c|\left(1+\frac{p(t+\tau+\delta)}{p(t+\delta)}\right)+\int_{t-\delta}^{t+\delta} p(s+\delta) d s\right\}<2 .
$$

together with (4) implies that every solution of the equation

$$
[x(t)-c x(t-\tau)]^{\prime}+p(t) x(t-\delta)=0, \quad t \geq t_{0}
$$

tends to zero as $t \rightarrow \infty$.

\section{Example}

Consider the difference equation

$$
\left[x(t)-\frac{t-1}{2 t} x(t-1)\right]^{\prime}+\frac{2}{(t-1)^{\alpha}}\left[1+\sin ^{2} x(t-2)\right] x(t-2)-\frac{1}{t^{\alpha}}\left[1+\sin ^{2} x(t-1)\right] x(t-1)=0,
$$

where $\alpha>0$ is a real constant, $f(x)=\left(1+\sin ^{2} x\right) x, p(t)=2 /(t-1)^{\alpha}, q(t)=1 / t^{\alpha}, c(t)=\frac{t-1}{2 t}$, $\tau=1, \delta=2, \sigma=1$. By simple estimation, we have

$$
\begin{gathered}
\mu=\lim _{t \rightarrow \infty}|c(t)|=\frac{1}{2}<1, \\
p^{*}(t)=p(t)-q(t+\sigma-\delta)=\frac{1}{(t-1)^{\alpha}},
\end{gathered}
$$




$$
2|x| \leq\left|\left(2+\sin ^{2} x\right) x\right| \leq 3|x|, \quad x^{2}\left(1+\sin ^{2} x\right)>0(x \neq 0)
$$

and

$$
\limsup _{t \rightarrow \infty}\left[\mu\left(1+\frac{p^{*}(t+\tau+\delta)}{2^{2} p^{*}(t+\delta)}\right)+\int_{t-\delta}^{t+\delta} p^{*}(s+\delta) d s+\frac{q(t+\sigma)}{p^{*}(t+\delta)} \int_{t-\delta}^{t} p^{*}(s+2 \delta) d s\right]=\frac{5}{8}<\frac{2}{3} .
$$

If $\alpha>0$, we may conclude from Theorems 1 and 2 that every solution of this equation is bounded and tends to a constant as $t \rightarrow \infty$; and if $0<\alpha \leq 1$, from Theorem 4 , every solution of this equation tends to zero as $t \rightarrow \infty$.

\section{Acknowledgement}

The author is grateful to the referee for helpful suggestions and comments.

\section{References}

[1] G. Ladas, Y. G. Sficas and I. P. Stavroulakis, Asymptotic behavior of solutions of retarded differential equations, Proc. Amer. Soc., 88(2) (1983), 247-253.

[2] K. Gopalsamy, Stability and Oscillation in Delay Differential Equations of Population Dynamics, Kluwer Academic Press, Boston, 1992.

[3] J. H. Shen and J. S. Yu, Asymptotic behavior of solutions of neutral differential equations with positive and negative coefficients, J. Math. Anal. Appl., 195(1995), 517-526.

[4] T. Yoneyama, On the 3/2 stability theorem for one-dimensional delay-differential equations with unbounded delay, J. Math. Anal. Appl., 165(1992), 133-143.

[5] J. W. H. So, J. S. Yu and M. P. Chen, Asymptotic stability for scalar delay differential equations, Funkcial. Ekvac., 39(1996), 1-17.

[6] G. Ladas and Y. G. Sficas, Asymptotic behavior of oscillatory solutions, J. Hiroshima Math., 18(1998), 351-359.

[7] X. H. Tang and X. F. Zou, Asymptotic stability of a neutral differential equation, Proc. Edinburgh Math. Soc., 45(2002), 333-347.

[8] J. S. Yu and B. G. Zhang, Stability theorem for delay differential equations with impulses, J. Math. Anal. Appl., 199(1996), 162-175.

[9] X. P. Wang and L. S. Liao, On the asymptotic behavior of solutions of a nonlinear difference-differential equation, Appli. Math. Lett., 18(2005), 267-272.

[10] G. P. Wei and J. H. Shen, Asymptotic behavior of solutions of nonlinear impulsive delay differential equations with positive and negative coefficients, Math. Comput. Modelling, 44(2006), 1089-1096.

[11] J. H. Shen, Y. J. Liu and J. L. Li, Asymptotic behavior of solutions of nonlinear neutral differential equations with impulses, J. Math. Anal. Appl., 332(1)(2007), 179-189.

[12] G. S. Ladde, V. Lakshimikantham and B. G. Zhang, Oscillation Theory of Differential Equations with Deviating Arguments, Marcel Dekker Press, New York, 1987.

[13] I. Gyori and G. Ladas, Oscillation Theory of Delay Differential Equations with Applications, Clarendon Press, Oxford, 1991.

Department of Mathematics, Huaihua College, Huaihua, Hunan 418008, China.

E-mail: weigengping@yahoo.com.cn 